\title{
Steady State Characteristics of Finite Hydrodynamic Porous Oil Journal Bearing Under Turbulent Regime
}

\author{
Kasturi Saikia $^{1}$, Kalyan Kalita ${ }^{2}$ \\ Research Student, Mechanical Engineering Department, Assam Engineering College, Guwahati, India ${ }^{1}$ \\ Associate Professor, Mechanical Engineering Department, Assam Engineering College, Guwahati, India ${ }^{2}$
}

\begin{abstract}
In this paper theoretical investigation of steady state characteristics of finite hydrodynamic porous oil journal bearing under turbulent regime has been conducted. Governing equation is solved numerically by using finite difference technique. Constantinescu's turbulent lubrication theory is used for this analysis. The steady state characteristics such as load carrying capacity, friction variable, end flow rate and attitude angle have been investigated over a broad span of variables like Reynolds's number, eccentricity ratio, slenderness ratio and bearing speed parameter. These characteristics are numerically evaluated using Borland C++ software for windows 7 operating system. The results are denoted by graphs which can be convenient for the analysis of such bearings.
\end{abstract}

Keywords: Porous bearing, hydrodynamic, turbulent, lubrication.

\section{INTRODUCTIUON}

The expeditious creative development of modern science and technology in the fields of electronics, nuclear engineering, computer science and space technology demands high speed bearing with high refinement working characteristics even in unfavorable working condition. Porous bearing are deliberated to provide hydrodynamic lubrication without the requirement of lubricant to supply externally. Porous bearings are widely used in industrial rate.

applications where other solid bearings are not practical to use due to the lack of space or unreachable lubrication, like domestic appliances, medical apparatus, computers, automobiles, machine tools, food and textile industry etc.

Turbulent has become practical importance in hydrodynamic lubrication, especially for high speed machinery. In an effort to determine the performance characteristics of finite porous oil journal bearing at high speed, turbulent Reynolds's equation for lubrication has been developed from the fundamental equation. In this paper this is done by assuming that porous journal bearing is full and running steady. Petroff [1] was first to consider the viscous friction in the fluid film bearings. The important contribution in the science of lubrication is the experiments of Tower [2] in 1883 and the work of Reynolds in 1886 , in which he made a review of detailed experimental [3] results of Tower. Taylor [4,5] studied theoretically flow between two concentric rotating cylinders and found the condition under which the laminar flow broke down to turbulent. An exhaustive study on the theory of turbulence in the lubrication has been given by Constantinescu's [6,7]. The effect of turbulence has been taken into account in the design of such bearings. A. Kumar and N.S Rao [8] originated the hydrodynamic theory of turbulent porous bearings. B.C Majumdar [9], described an analytical solution of hydrostatic porous journal bearing. K. Kalita [10], theoretically investigated steady state and dynamic characteristics of hybrid porous

oil journal bearing under turbulent regime. B.C Majumdar and A.K Chattopaudhyay [11] have presented a theoretical analysis and experimental investigation of steady state characteristics of externally pressurized porous oil journal bearings. The purpose of the present analysis is to study the steady state behavior in terms of load carrying capacity, friction variable, and attitude angle and oil flow

\section{MATHEMATICAL FORMULATION}

A schematic diagram of hydrodynamic porous journal bearing is shown in fig 1 . The journal of radius $R$ operating with steady state eccentricity ratio $\epsilon_{0}$ rotates with a uniform tangential velocity $U$. The wall thickness $H$ is fitted in a solid housing. The pores of the bearings are impregnated with incompressible Newtonian fluid of constant viscosity. Pressurized oil with a supply pressure $P_{s}$ is fed to the outer surface of the bearing. To obtain the governing equation of flow through porous matrix, Darcy's law is implemented. The Cartesian co-ordinate system is used for the analysis of the fluid film in the bearing clearance space and it is considered that oil film in the bearing clearance space is turbulent.

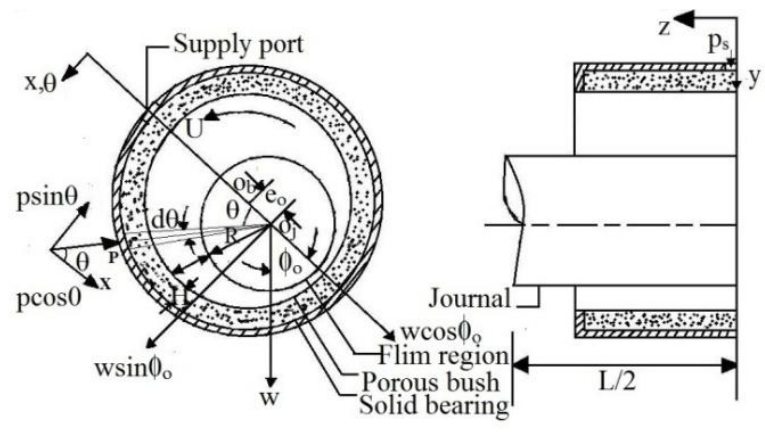

Fig 1 Hydrodynamic porous journal bearing under steady state condition 
The generalized steady state equation of flow in the theory of hydrodynamic lubrication and the turbulent Reynolds's equation for porous matrix can be written as

$\emptyset_{\mathrm{x}}\left(\frac{\partial^{2} \mathrm{p}_{0}}{\partial \mathrm{x}^{2}}\right)+\emptyset_{\mathrm{y}}\left(\frac{\partial^{2} \mathrm{p}_{0}}{\partial \mathrm{y}^{2}}\right)+\emptyset_{\mathrm{z}}\left(\frac{\partial^{2} \mathrm{p}_{0}{ }_{0}}{\partial \mathrm{z}^{2}}\right)=0$

$\frac{\partial}{\partial x}\left(\frac{\frac{h_{o}{ }^{3}}{\mu k_{x}} \partial p_{o}}{\partial x}\right)+\frac{\partial}{\partial z}\left(\frac{\frac{h_{o}{ }^{3}}{\mu k_{z}} \partial p_{o}}{\partial z}\right)=\frac{U}{2} \frac{\partial h_{o}}{\partial x}+\frac{\partial h}{\partial t}+\frac{\varphi_{\mathrm{y}}}{\mu}\left(\frac{\partial \mathrm{p}_{o}^{\prime}}{\partial \mathrm{y}}\right)_{\mathrm{y}=0}$

$k_{x}$, and $k_{z}$, the turbulence co-efficients. Inserting the dimensionless parameters given in the following in Equations (1) and (2), we get,

$\overline{p^{\prime}}{ }_{o}=\frac{p_{o}^{\prime}}{p_{s}}, \bar{y}=\frac{y}{H}, \overline{p_{o}}=\frac{p_{o}}{p_{s}}, \overline{h_{o}}=\frac{h_{o}}{C}, \bar{z}=\frac{2 z}{L}, \theta=\frac{x}{R}, \overline{p^{\prime}}=$ $\frac{p^{\prime} C^{2}}{\mu U R}, \bar{p}=\frac{p C^{2}}{\mu U R}, \overline{\varphi_{x}}=\frac{\varphi_{x}}{\varphi_{y}}, \overline{\varphi_{z}}=\frac{\varphi_{z}}{\varphi_{y}}, \lambda=\frac{\mu U R}{C^{2} p_{s}}, \beta=\frac{12 \varphi_{y} R^{2}}{C^{3} H}$

$\frac{\varphi_{x} \partial^{2} \overline{p_{o}^{\prime}}}{\partial \theta^{2}}+\frac{\partial^{2} \overline{p^{\prime}{ }_{o}}}{\partial \bar{y}^{2}}\left(\frac{R^{2}}{H^{2}}\right)+\frac{\varphi_{z} \partial^{2} \overline{p^{\prime}{ }_{o}}}{\partial \bar{z}^{2}}\left(\frac{D}{L}\right)^{2}=0$

$\frac{\partial^{2} p_{o}}{\partial \theta^{2}}\left(\overline{\frac{h^{3} o}{k_{x}}}\right)+\left(\frac{D}{L}\right)^{2} \frac{\partial^{2} \overline{p_{o}}}{\partial \bar{z}}\left(\overline{\frac{h^{3} o}{k_{x}}}\right)=\frac{\lambda}{12}\left(\frac{\partial \overline{h_{o}}}{\partial \theta}\right)+\frac{\beta}{12} \overline{p_{o}}-\frac{\beta}{12}$

Where the turbulence co-efficients $k_{x}$ and $k_{z}$ are is given by,

$k_{x}=12+0.026(R e *)^{0.8265}$

$k_{z}=12+0.0198(R e *)^{0.741}$

$k_{z}=12+0.0198(R e *)$
Here $\overline{h_{o}}$ and $k_{x}$ are functions of $\theta$ only and $k_{z} f\left(\frac{R}{C}\right)=\frac{\overline{F_{s}}}{2 \bar{w}}, f$ is co-efficient of friction.

independent of $z$.

\section{BOUNDARY CONDITION}

The boundary conditions in the film region are given below:

The pressure is symmetrical about the origin in $\mathrm{Z}$ directions,

$\frac{\partial \overline{p_{o}}}{\partial \bar{z}}(\theta, 0)=0$

The bearing ends are considered to be open to the atmosphere,

$\overline{p_{o}}(\theta, \pm 1)=0$

The Swift-Stieber condition or Reynolds's boundary condition is,

$\left(\frac{\partial \overline{p_{o}}}{\partial \bar{\theta}}\right)_{\theta=\theta_{2}}=0$ for $\left(\theta_{2} \leq \theta \leq 2 \pi\right)$

Where $\theta_{2}$ be the angle at which the film cavitates.

Again,

$\overline{p_{o}}(\theta, \bar{z})=\overline{p_{o}}(\theta+2 \pi, \bar{z})$

Whenever the pressures become negative at any mesh point in the iteration, it should be made equal to zero or the cavitations' pressure, i.e. $\overline{p_{o}}=0$ If $\overline{p_{o}}<0$, in the cavitations zone, $\theta \geq \theta_{2}$.

\section{ANALYSIS OF STEADY STATE CHARACTERISTICS}

A. Load Carrying Capacity:

The load carrying capacity can be evaluated by integrating the following pressure along and perpendicular to the line of center, i.e.

$\overline{w_{x}}=-\frac{1}{2} \int_{0}^{1} \int_{0}^{\theta_{2}} \overline{p_{o}} \cos \theta R d \theta d \bar{z}$

$\overline{w_{z}}=\frac{1}{2} \int_{0}^{1} \int_{0}^{\theta_{2}} \overline{p_{o}} \sin \theta R d \theta d \bar{z}$
Where $w_{x}$ and $w_{z}$ are the components of load carrying capacity along and perpendicular to the line of centers respectively.

The load parameter is given by,

$\bar{w}=\left(\overline{w_{x}^{2}}+\overline{w_{z}^{2}}\right)^{\frac{1}{2}}$

And attitude angle is given by,

$\varphi_{0}=\tan ^{-1}\left(\frac{\overline{w_{z}}}{\overline{w_{x}}}\right)$

B. Friction Variable:

Shearing stress acting on the surface of the journal can be represented by [13]

$\tau=\frac{\mu U}{h} \overline{\tau_{c}}+\frac{h}{2}\left(\frac{\partial p}{\partial x}\right)$

Where $\overline{\tau_{c}}=1+0.0023\left\{\operatorname{Re} \cdot \overline{\left(h_{o}\right)}\right\}^{0.855}$

The equation (7) is valid for non-cavitated zone and total friction force in the non-cavitated zone is given by,

$\overline{F_{s 1}}=2 \int_{0}^{1} \int_{0}^{\theta_{2}}\left\{\frac{\mu U}{h} \overline{\tau_{c}}+\frac{h}{2}\left(\frac{\partial p}{\partial x}\right)\right\} R d \theta d z$

Now frictional force for the cavitated zone can be calculated as suggested by [12] and obtained as

$\overline{F_{s 2}}=\overline{h_{2}} \int_{0}^{1} \int_{\theta_{2}}^{2 \pi} \frac{\lambda \overline{\tau_{c}}}{6 \overline{h_{o}}} d \theta d \bar{z}$

Where $\overline{h_{2}}=1+\epsilon_{0} \cos \theta_{2}$

Thus the total frictional force is

$\overline{F_{s}}=\overline{F_{s 1}}+\overline{F_{s 2}}$

And friction variable is given by,

The equation (7) is valid for non-cavitated zone and total friction force in the non-cavitated zone is given by,

$\overline{F_{s 1}}=2 \int_{0}^{1} \int_{0}^{\theta_{2}}\left\{\frac{\mu U}{h} \overline{\tau_{c}}+\frac{h}{2}\left(\frac{\partial p}{\partial x}\right)\right\} R d \theta d z$

Now frictional force for the cavitated zone can be calculated as suggested by [12] and obtained as

$\overline{F_{s 2}}=\overline{h_{2}} \int_{0}^{1} \int_{\theta_{2}}^{2 \pi} \frac{\lambda \overline{\tau_{c}}}{6 \overline{h_{o}}} d \theta d \bar{z}$

Where $\overline{h_{2}}=1+\epsilon_{0} \cos \theta_{2}$

Thus the total frictional force is

$\overline{F_{s}}=\overline{F_{s 1}}+\overline{F_{s 2}}$

And friction variable is given by,

$f\left(\frac{R}{C}\right)=\frac{\overline{F_{S}}}{2 \bar{w}}, f$ is co-efficient of friction.

C. Oil Flow Rate:

The oil can be flowed from the bearing by the following two ways

(a) Flow from the open ends of the bearing

(b) Flow from the clearance space of the bearing

At the open ends of the bearing bush, flow is negligible as compared to clearance space. Hence only flow from the clearance space is calculated.

Flow through the clearance space is given by,

$\bar{Q}=-\frac{1}{3} \int_{0}^{2 \pi} \frac{\partial \overline{p_{o}}}{\partial z} \overline{h^{3}{ }_{o}} d \theta$

$\bar{W}, \overline{F_{S}}$ and $\bar{Q}$ Values are calculated adopting the Trapezoidal rule of numerical integration.

\section{RESULTS AND DISCUSSION}

A computer program in $\mathrm{C}$ using the relaxation iterative method to solve the governing equation is developed. The program could consider the laminar flow condition by letting $\beta=0$ and by allowing $k_{x}=k_{x}=12$ with $R e=0$

(6) in the generalized program and also for laminar flow the 
value of $\overline{\tau_{c}}$ was put equal to unity. The results obtained by computer program operating in turbulent flow regime were generated. The steady state performance or characteristics of bearings depends on the parameters viz. $\beta, R e, L / D, \lambda$ and $\epsilon_{0}$. In the present analysis the effect of various parameters on load carrying capacity, friction variable, oil flow rate and attitude angle are analyzed by varying all the above mentioned parameters.

\section{A. Effect of $\beta$ and Re}

The effect of the bearing feeding parameter shows that the load carrying capacity decreases (Fig 2) and friction coefficient increases (Fig 3). For lower values of $\beta, \bar{w}$ remains unaffected by a change of $\beta$. However lower values of $\beta$ depends upon Re. It is seen that for various values of $R e$ friction variable remains constants for lower values of feed parameter $(\beta \leq 0.1)$ and for higher values of $\beta$ friction variable increases. Again Re increases oil flow rate increases (Fig 4). For all values of $R e$, oil flow rate remains constant up to certain values of $\beta(\beta \leq 0.1)$ and then increases for higher values of $\beta$. The attitude angle is more prominent for lower

values of $\beta$.

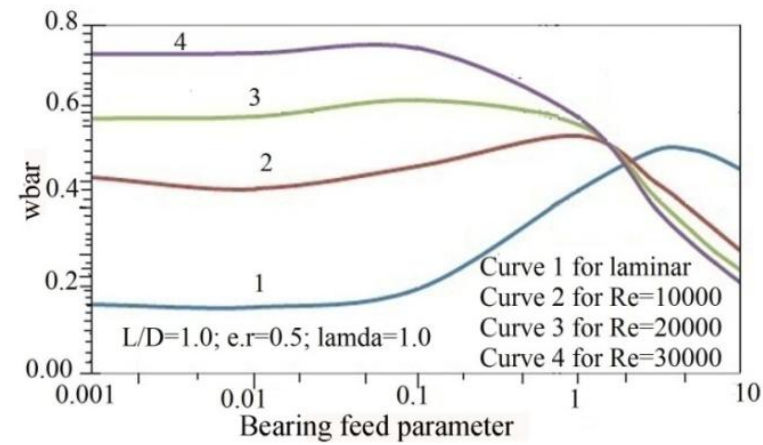

Fig 2 Non-dimensional load capacity VS beta for different Re

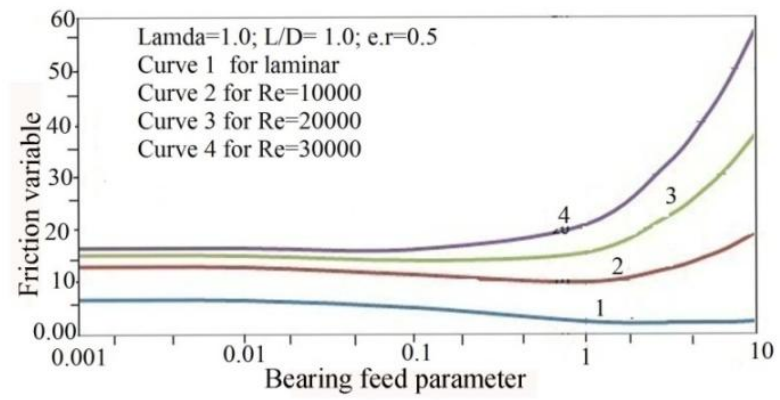

Fig 3 Friction variable VS beta for different Re

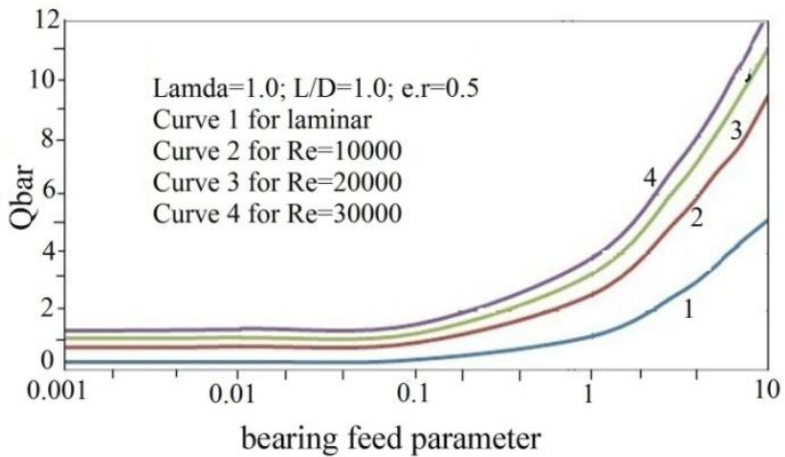

Fig 4 Oil flow rate VS beta for different Re

\section{B. Effect of $L / D$}

The effect of $\mathrm{L} / \mathrm{D}$ ratio is to increase the load carrying capacity (Fig 5) and decrease the friction variable (Fig 6). However for particular higher values of $\mathrm{L} / \mathrm{D}(\mathrm{L} / \mathrm{D} \geq 2) \bar{w}$ decreases. The oil flow rate and attitude angle also increases with the increasing L/D ratio (Fig 7 and 8 ).

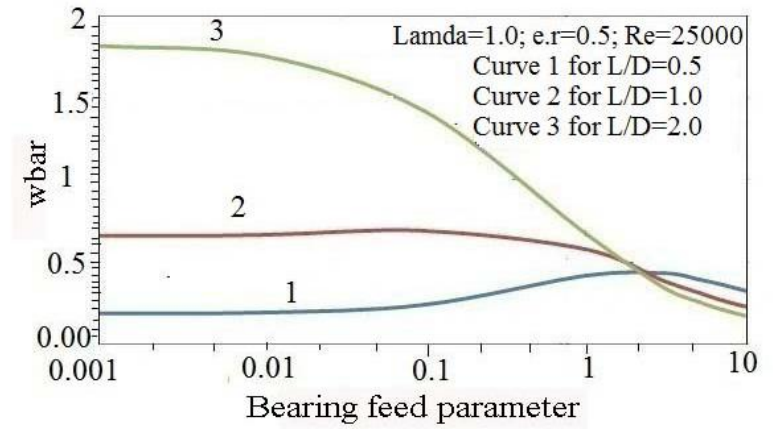

Fig 5 Load carrying capacity vs beta for different L/D ratio

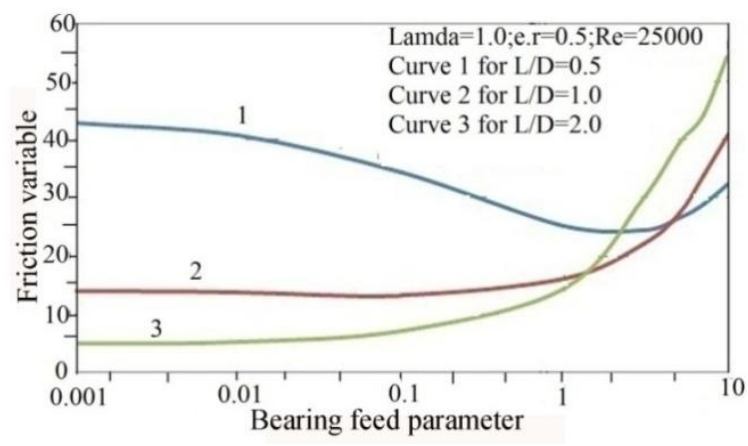

Fig 6 Friction variable VS beta for different L/D ratio

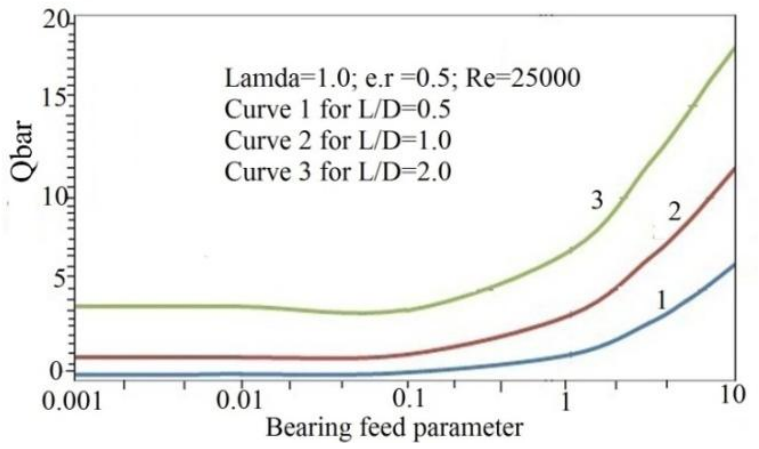

Fig 7 Oil flow rate VS beta for different L/D ratio

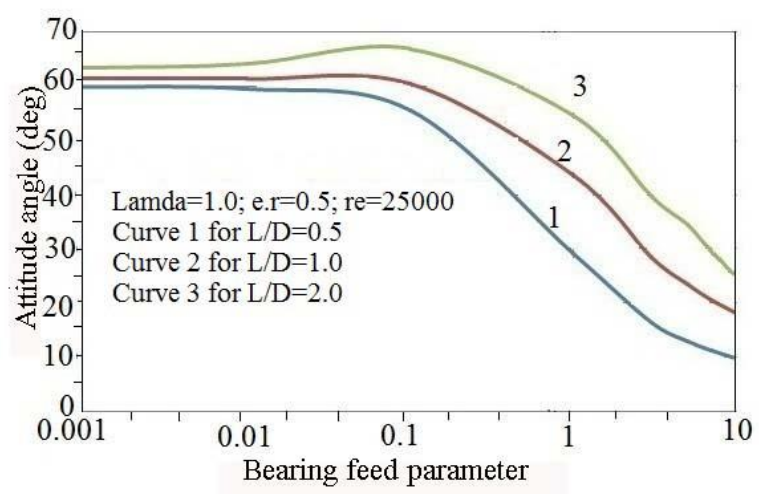

Fig 8 Attitude angle VS beta for different L/D ratio

C. Effect of $\lambda$

Load carrying capacity increases as speed parameter increases for a certain range of $\beta$ (Fig 9). However at 
higher values of $\lambda(\cong 10), \bar{w}$ decreases at a faster rate with $\beta$. Friction variable increases as $\lambda$ increases (fig 10). Oil flow rate increases as $\lambda$ increases up to a certain values of $\beta$ (fig 11). Further increase of $\beta$, they have found to be merged with each other.

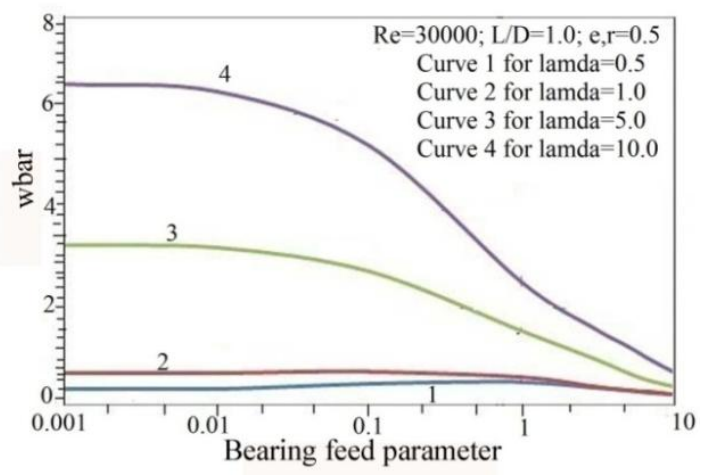

Fig 9 Load carrying capacity VS beta for different lamda

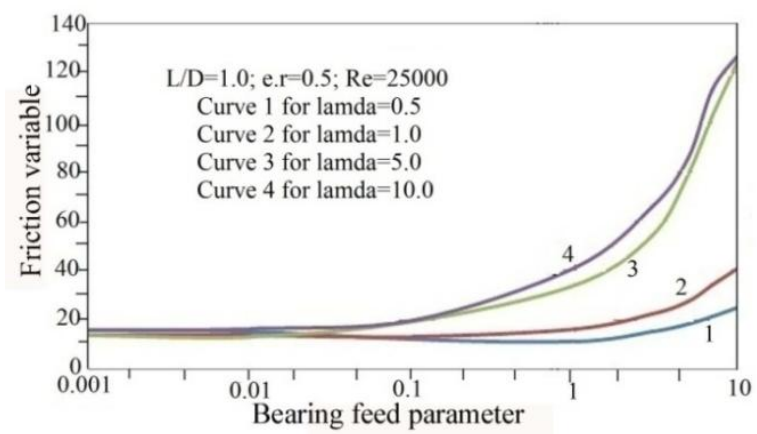

Fig 10 Friction Variable VS beta for different lamda

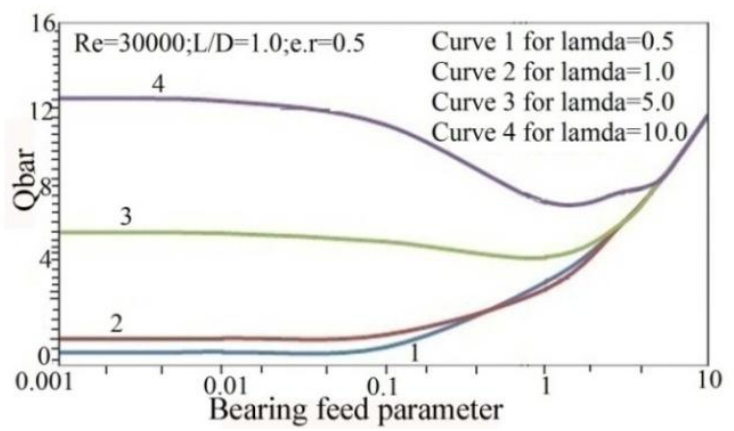

Fig 11 Oil flow rate VS beta for different lamda

\section{Effect of $\in_{0}$ :}

The effect of eccentricity ratio is to increase the load capacity and to decrease the friction variable (Fig 12 and 13).

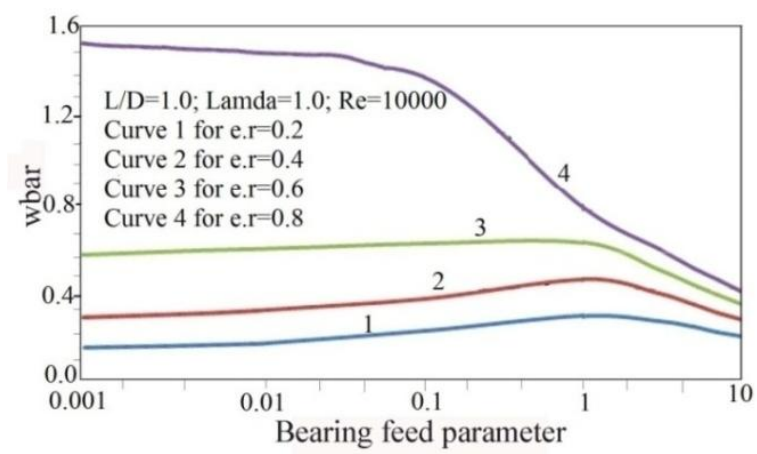

Fig 12 Load carrying capacity VS beta for different eccentricity ratio

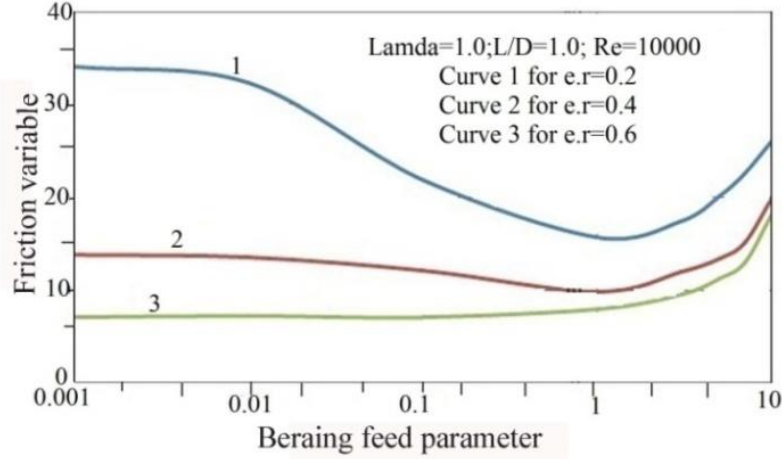

Fig 13 Friction variable Vs beta for different eccentricity ratio

With the increase in eccentricity ratio oil flow rate increases up to a certain value of $\beta$ (Fig 14), beyond which flow rate is almost same. Attitude angle decreases as eccentricity ratio increases (Fig 15).

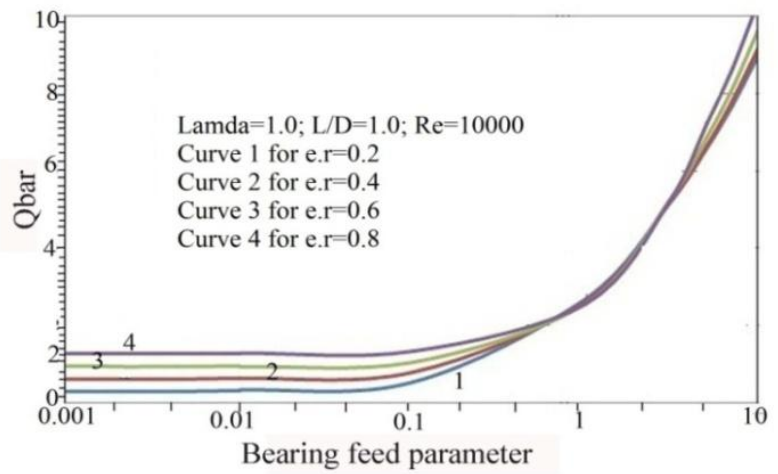

Fig 14 Oil flow rate VS beta for different eccentricity ratio

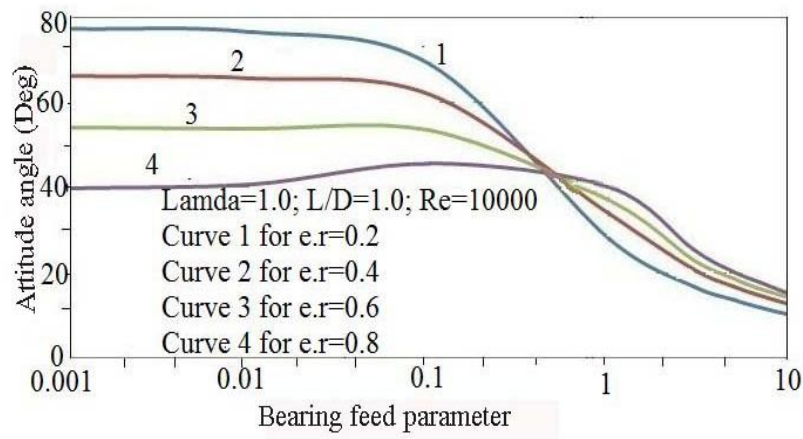

Fig 15Attitude angle VS beta for different eccentricity ratio

\section{CONCLUSION}

The effect of various parameters on steady state parameters have been investigated theoretically in a finite porous oil journal bearing on the basis of Constantinescu's theory of turbulent lubrication. The pressure distribution equation is solved numerically with the help of finite difference technique with a grid having 44 divisions in $\theta$ direction and 7 divisions in $z$ direction.

On the basis of the results presented in fig 2-11, the following conclusion can be found.

1. The effect turbulence as Re increases is to increase the load carrying capacity, friction variable, and oil flow rate and attitude angle. But at higher values of $\beta,(\beta>2)$, load carrying capacity decreases sharply. 
2. Oil flow rate increases as Reynolds's number, speed $p$ parameter or eccentricity ratio increases. But at higher $\bar{p}$ values of the curves tend to merge with each other.

3. The increase in non dimensional load carrying capacity $\left(\mathrm{N} / \mathrm{m}^{2}\right)$ is more accentuated for lager values of eccentricity ratio $\overline{p_{o}}$ parameters.

Thus the bearing feed parameter value should be $p^{\prime}$ maintained as low as possible, ideally around 0.1 , for better a pursuance of turbulent bearings.

\section{NOMENCLATURE}

C Radial clearance (m)

$e \quad$ Eccentricity (m)

$D \quad$ Diameter of journal (m)

$\mu \quad$ Co-efficient of viscosity of the fluid (pas)

$\emptyset \quad$ Attitude angle (rad. or degree)

$f \quad$ Coefficient of friction

$f\left(\frac{R}{c}\right) \quad$ Friction variable.

$F_{S} \quad$ Friction force $(\mathrm{N})$

$F_{s 1} \quad$ Friction force in non-cavitated region $(\mathrm{N})$

$F_{s 2} \quad$ Friction force in cavitated region $(\mathrm{N})$

$\frac{F_{s 1}}{F_{s}} \quad$ Non-dimensional friction force in non-cavitated region $(\mathrm{N})$

$\overline{F_{s 2}} \quad$ Non-dimensional friction force in cavitated region $(\mathrm{N})$

$h \quad$ Local fluid film thickness (m)

$\bar{h} \quad$ Non-dimensional fluid film thickness

$h_{o} \quad$ Steady State local fluid film thickness (m)

$\overline{h_{o}} \quad$ Non-dimensional local steady state fluid film thickness

$h_{2} \quad$ Fluid film thickness where cavitations starts (m)

$\overline{h_{o}} \quad$ Non- dimensional form of $h_{2}$

$H \quad$ Thickness of the wall of the porous bush (m)

$w \quad$ Load carrying capacity of the bearing $(\mathrm{N})$

$\bar{W} \quad$ Non-dimensional load capacity of the

bearing

$w_{x}, w_{z}$ Components of load carrying capacity along and perpendicular to the line of centers

$\overline{w_{x}}, \overline{w_{z}} \quad$ Non-dimensional form of $W_{x}$ and $W_{z}$

$x, y, z \quad$ Circumferential, radial and axial co-ordinates

$\rho \quad$ Density of the lubricating film $\left(\mathrm{kg} / \mathrm{m}^{3}\right)$

$\tau \quad$ Shear stress on journal surface $\left(\mathrm{N} / \mathrm{m}^{2}\right)$

$\varphi_{0} \quad$ Steady state attitude angle (Rad. or degree)

$\varphi_{x}, \varphi_{y}, \varphi_{z}$ Permeability coefficients of porous medium

along $\mathrm{x}, \mathrm{y}$ and $\mathrm{z}$ direction $\left(\mathrm{m}^{2}\right)$

$\in \quad$ Eccentricity ratio

$\epsilon_{0} \quad$ Steady state eccentricity ratio

$\lambda \quad$ speed parameter which is equal to $\frac{\mu U R}{C^{2} p_{s}}$

$C \beta \quad$ Bearing feed parameter which is equal to $\frac{12 \varphi_{y R^{2}}}{C^{3} H}$

$\theta_{2} \quad$ Angle at which the fluid film starts to cavitates

$\Delta \theta, \Delta z$ Finite difference meshes size along circumference and length of the bearing. (m)

$k \quad$ Constants

$k_{x}, k_{z} \quad$ Constants in turbulent Reynolds equation

$l \quad$ Length of the bearing (m)

$L / D \quad$ Slenderness ration or Aspects ratio

$n \quad$ Number of iterations
Pressure in the clearance space $\left(\mathrm{N} / \mathrm{m}^{2}\right)$

Non-dimensional form of $p$

Steady state pressure in the clearance space

Non dimensional form of $p_{o}$

Pressure in the porous medium (pa)

Non-dimensional form of $p^{\prime}$

Steady state pressure in the porous medium (pa)

Non-dimensional form of $\mathrm{p}^{\prime}$ 。

$p_{s} \quad$ Oil supply pressure to the bearing $(\mathrm{pa})$

$u, v, w$ Velocity component along $\mathrm{x}, \mathrm{y}$ and $\mathrm{z}$

directions respectively in the fluid film region $(\mathrm{m} / \mathrm{s})$

$u^{\prime}, v^{\prime}, w^{\prime}$ Velocity component along $\mathrm{x}, \mathrm{y}$ and $\mathrm{z}$ directions

respectively in the porous medium $(\mathrm{m} / \mathrm{s})$

$U \quad$ Surface velocity of journal $(\mathrm{m} / \mathrm{s})$

$Q \quad$ End flow of oil $\left(\mathrm{m}^{3} / \mathrm{s}\right)$

$\bar{Q} \quad$ Non-dimensional form of $Q$

$R \quad$ Radius of the journal (m)

$R e \quad$ Average Reynolds number which is equal to $\frac{\rho U C}{\mu}$

$R e * \quad$ Instantaneous Reynolds number which is equal to

$\frac{\rho U h}{\mu}$

$R^{\prime}$ Modified Reynolds number which is equal to $k^{2} R e *$

$\tau_{c}$ Coquette surface shear stress $\left(\mathrm{N} / \mathrm{m}^{2}\right)$

$\overline{\tau_{c}}$ Non dimensional form of $\tau_{c}$ which is equal to $\frac{\tau_{c} h}{\mu U}$

$\bar{y}$ Dimensional Radial co-ordinate which is equal to $\frac{y}{H}$

$\bar{z}$ Dimensional axial coordinate which is equal to $\frac{2 z}{L}$

\section{REFERENCES}

1. Petroff, N. (1883), Friction in machines and the effect of the lubricant (in Russian ) J. St. petersberg, 1-4, 71, 228, 377 and 530.

2. Tower, B. First report on friction experiments Proc. Inst. Of Mech. Engg., London, No. 34, 1883

3. Tower, B. Second report on friction experiments Proc. Inst. Of Mech. Engg., London, No. 34, 1883

4. Taylor, G.I (1923), Stability of a viscous liquid contained between two rotating cylinders, Trans. Roy.Sco, London 223 A, 289-343.

5. Taylor, G.I (1936), Fluid friction between rotating cylinders, Proc. Roy.Soc, London, A 157,546.

6. Constantinescu, V.N (1958), Influence of turbulence on the motion in the lubricating layer, Studii si cer. De Mec Aplicata, Accd. Rep. Pop. Romie, 60, 103-162.

7. Constantinescu, V.N (1962), Analysis of bearings operating in turbulent regimes, J. Basic Engg, Trans. ASME, D, 84, 139-151.

8. A. Kumar Mech. Engg department, Regional Institute of Technology, Jamshedpur , N.S. Rao, Mech. Engg Department, Indian Institute of Technology, Kharagpur Steady state performance of finite hydrodynamic porous journal bearings in turbulent regime wear, 167, pp 121-126, 1993.

9. B.C Majumdar, "Introduction to Tribology of bearings", a.h Wheeler and co. private limited, edition 1986.

10. Kalyan Kalita, Steady state and dynamic characteristics of hybrid oil journal bearing with porous bush under turbulent regime, Dept. of Mech. Engg, IIT kharagpur, December 1991.

11. B.C Majumdar, and A.K. Chattopadhyay, Steady State solution of finite hydrostatic porous oil bearings with tangential velocity slip, Tribology International, Dec. 1984, pp. 317-323. 17. Floberg, L. Boundary Conditions of cavitation regions in journal bearings ASLE. Trans, 4, 1961, pp. 282-286

12. Floberg, L. "Boundary Conditions of cavitation regions in journal bearings" ASLE. Trans, 4, 1961, pp. 282-286.

13. Constantinescu, V.N and Galetuse, S. On the determination of friction forces in turbulent lubrication, ASLE.Trans.vol8, 1965, pp.367-380 